\title{
Clinical Study \\ Successful Treatment in Children with Hodgkin Lymphoma in Greece; A 20-Year Experience in a Single Institution
}

\author{
Apostolos Pourtsidis, ${ }^{1}$ Dimitrios Doganis, ${ }^{1}$ Margarita Baka, ${ }^{1}$ Despina Bouhoutsou, ${ }^{1}$ \\ Maria Varvoutsi, ${ }^{1}$ Maria Synodinou, ${ }^{2}$ Katerina Strantzia, ${ }^{3}$ and Helen Kosmidis ${ }^{1}$ \\ ${ }^{1}$ Oncology Department, Lab of Children's Hospital P \& A. Kyriakou, Thivon \& Levadias Street, 11527 Athens, Greece \\ ${ }^{2}$ Radiation Department, Lab of Children's Hospital P \& A. Kyriakou, Thivon \& Levadias Street, 11527 Athens, Greece \\ ${ }^{3}$ Pathology Department, Lab of Children's Hospital P \& A. Kyriakou, Thivon \& Levadias Street, 11527 Athens, Greece
}

Correspondence should be addressed to Apostolos Pourtsidis, tolisp@otenet.gr

Received 24 May 2012; Revised 30 July 2012; Accepted 22 August 2012

Academic Editor: Stefano Luminari

Copyright (C) 2012 Apostolos Pourtsidis et al. This is an open access article distributed under the Creative Commons Attribution License, which permits unrestricted use, distribution, and reproduction in any medium, provided the original work is properly cited.

\begin{abstract}
During the last 30 years, combined chemotherapy regimens with radiotherapy or not significantly improved the prognosis for patients with Hodgkin lymphoma. We retrospectively studied 58 children (35 boys and 23 girls) with Hodgkin lymphoma who were treated at our institution during the period 1987-2006 and we correlated age, sex, stage, histology, and therapy with the outcome of patients. Of our patients, 9 children were 8 years old or younger. Nodular sclerosis was the predominant histology subtype (69\%), whereas 26 patients $(45 \%)$ had advanced disease (stage III or IV). Chemotherapy (CT) with various drug combinations, according to the period of treatment plus low-dose involved field radiation therapy (IFRT), was used in all patients. Five children experienced relapse and in 3 other patients second or third malignancies were documented. The overall survival was found to be $98 \%$. No factors related to the outcome could be detected. The prognosis of children with Hodgkin lymphoma is excellent with CT combined with low dose IFRT but in long-time survivors late effects of the combined modality treatment are still issues of major concern. Longer followup of a greater number of patients is necessary to detect prognostic factors related to the outcome of children with Hodgkin lymphoma and to identify some patients who would be treated without radiation.
\end{abstract}

\section{Introduction}

In Europe and USA Hodgkin lymphoma (HL) is the sixth most common type of cancer in children following leukemia, CNS tumors, neuroblastoma, nephroblastoma, and non Hodgkin lymphomas, accounting for approximately $5 \%$ of all childhood malignancies. The prevalence of disease varies considerably with age, gender, nationality, and histology subtype. According to data of International Agency for Research on Cancer (IARC) for children younger than 15 years, the incidence is 5.5 cases per million but for those aged 15-20 years the incidence reaches 12.1 cases per million. In Western Asia (extending from the Mediterranean to Northwest India) the incidence in children $<15$ years old is consistently higher than 7 cases per million [1]. In Greece, the annual incidence is 7.2 per million children [2]. The disease presents a characteristically bimodal curve with an early peak in adolescence and a second peak in adults aged above 50 years old, whereas is very rare in children under 5 years of age. Patients (pts) with HL commonly present with cervical or supraclavicular lymphadenopathy and with mediastinal involvement in most of them. Treatment is largely determined by disease stage, patient's age at diagnosis, the presence or absence of " $\mathrm{B}$ " symptoms, the presence of bulky nodal disease, and the rapidity of response to therapy [3]. During the last 20 years the introduction of combined chemotherapy (CT) protocols with or without radiotherapy (RT) improved significantly the outcome of children with HL. The 5-year event-free survival (EFS) in childhood and adolescence exceeds $90 \%$ for patients with early-stage disease [4] and 70 to $80 \%$ for those with advanced stage $[5,6]$. However, several pts mainly with advanced stage still relapse and in some the outcome is dismal. The purpose of our retrospective study was to report the treatment results, 
TABLE 1: Epidemiologic and laboratory findings of patients.

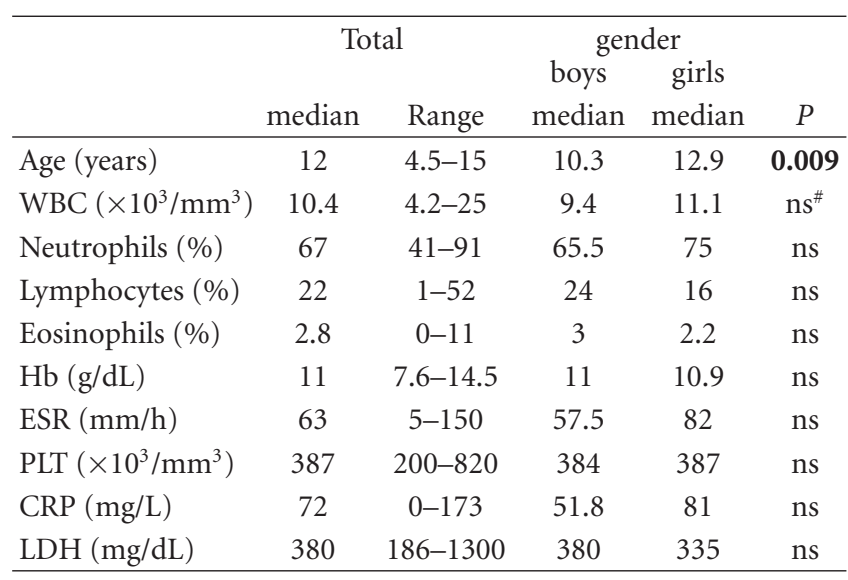

\#non-significant.

prognostic, factors, and final outcome of childhood pts less than 15 years of age with HL treated with CT followed by low-dose RT from January 1987 to December 2006 in the Oncology Department of Panagiotis and Aglaia Kyriakou Children's Hospital of Athens, Greece.

\section{Material and Methods}

A retrospective study of children diagnosed as suffering from Hodgkin Lymphoma from January 1, 1987 to December 31, 2006, was conducted in the Oncology Department of our hospital. All children underwent physical examination, blood testing, and imaging studies (X-ray, sonography, computed tomography, and magnetic resonance imaging) as clinically indicated. Staging was carried out according to the Ann Arbor system [7], whereas histopathology subtypes were defined according to Rye classification system [8].

From 1987 to 1993 our patients were treated according to French Society of Paediatric Oncology protocol MDH82 [9]: those with early stage (stage I or IIA) received four cycles of Doxorubicin, Bleomycin, Vinblastine, Dacarbazine (ABVD) plus radiotherapy (20 Gy) in initially involved fields, whereas those with advanced stage IIB, III, and IV were treated with alternating three cycles of Mustine, Vincristine, Procarbazine, Prednisone (MOPP)/ABVD plus radiotherapy (20 Gy) in initially involved fields plus spleen and paraaortic lymph nodes. During the second period (1994 to 2006) our patients were treated as follows: pts with early stage (stage I or II) received four cycles of Vinblastine, Bleomycin, Etoposide, and Prednisone (VBVP) plus radiotherapy (20 Gy) in initially involved fields according to French Society of Pediatric Oncology protocol MDH90 study [10]. Pts with stage III were treated with alternating two cycles of MOPP/ABVP plus radiotherapy $(20 \mathrm{~Gy})$ in initially involved fields, spleen, and paraaortic lymph nodes. Pts with stage IV received 2 cycles of Vincristine, Prednisone, Procarbazine, and Doxorubicin (OPPA), and four cycles of Cyclophosphamide, Vincristine, Procarbazine, Prednisone (COPP) plus radiotherapy (20 Gy) as above in initially involved lymph nodes and organs. Especially for stage IV disease, pts received 12 Gy to the liver and 20-30 Gy to the bones for active disease at diagnosis. Also, lungs were irradiated $(12 \mathrm{~Gy})$ only if there was no complete lung remission following two cycles of OPPA. In all, treatment response was assessed by imaging initially involved areas after completion of the fourth cycle of chemotherapy. A good response was defined as either complete remission or more than a $70 \%$ reduction in tumour size. Good responders received 20 Gy of RT to initially involved fields. The remaining (poor responders) were given one or two cycles of OPPA. Poor responders to OPPA received radiotherapy in dose of $40 \mathrm{~Gy}$. In all cases RT fields were limited to involved sites, as identified by the initial physical examination and imaging.

Epidemiologic, clinical, laboratory, and imaging findings at diagnosis as well as the treatment modalities were analyzed. Prognostic factors studied were age, sex, stage, B symptoms (present versus absent), hemoglobin $(<10.5 \mathrm{~g} / \mathrm{dL}$ versus $>10.5 \mathrm{~g} / \mathrm{dL}$ ), histology, and bulky mediastinal disease (Mediastinum/Thorax ratio $>1 / 3$ versus $<1 / 3$ ). Overall, event- or disease-free survival was estimated with KaplanMeier's curves. As events, death, relapse, and second malignancy were defined. For statistical analysis the following tests were used: (1) nonparametric Mann-Witney test, (2) chisquare test, (3) fisher's exact test. The study was approved by the local Institutional Research and Ethics Committee.

\section{Results and Outcome}

During the study period 58 children ( 35 boys and 23 girls at a $1.5 / 1$ ratio) were treated in our institution. Age at diagnosis ranged between 4.5 to 15 years (median 12 years). Three were 5 years old or younger and all aged 7 years or less (7 pts) were boys. Main laboratory findings of our pts at diagnosis are shown in Table 1. The time interval from first clinical symptom/sign until diagnosis ranged from 3 days to 7 months (median 1.5 months) without any correlation with gender and age. The most common presenting symptom at diagnosis was lymphadenopathy (44 patients, $76 \%$ ), whereas in 4 patients HL was diagnosed during investigation for fever, 2 for chronic cough, 3 during random imaging examination, and 5 during evaluation for other symptoms or signs. In two children the diagnosis was established during investigation for nephrotic syndrome which was a paraneoplastic manifestation of HL. In 21 pts (36.2\%) apart from the main symptom/sign the initial clinical presentation included cough, pleurodynia, fever, lymphadenopathy, dyspnea, or a combination of them. B symptoms were present in 18 pts $(31 \%)$ mainly in nodular sclerosis subtype $12 / 40$ (30\%). Moreover, B symptoms were detected in $6 / 25(24 \%)$ of stage II pts, in $9 / 15$ (60\%) of stage III, and in $3 / 11(27 \%)$ of stage IV $(P=0.028)$.

3.1. Imaging Investigations. Computed tomography of the neck, chest X-ray, and chest computed tomography were abnormal in $84 \%, 67 \%$, and $76 \%$ of pts, respectively. Abdominal ultrasound and computed tomography detected abnormal findings in $39 \%$ and $37.5 \%$ pts, respectively. Hepatomegaly was detected in 6, splenomegaly in 13 , and 
TABLE 2: Epidemiology and laboratory findings of patients and stage and histology of the disease.

\begin{tabular}{|c|c|c|c|c|c|c|c|c|c|}
\hline & \multicolumn{5}{|c|}{ Stage } & \multicolumn{4}{|c|}{ Histology*** } \\
\hline & $\mathrm{I}$ & II & III & IV & $P$ & $\mathrm{MC}$ & $\mathrm{LD}$ & LP & NS \\
\hline Gender (boys : girls) & $4: 3^{*}$ & $14: 11$ & $10: 5$ & $7: 4$ & $\mathrm{~ns}^{\#}$ & $12: 1$ & $1: 0$ & $4: 0$ & $18: 22$ \\
\hline Age (years) & $9.8^{* *}$ & 12.1 & 12.3 & 12.9 & ns & 10.2 & 10.2 & 10.5 & 12.5 \\
\hline $\mathrm{WBC}\left(\times 10^{3} / \mathrm{mm}^{3}\right)$ & 7.3 & 11.68 & 9.5 & 10.8 & 0.026 & 7.2 & 4.7 & 7.05 & 11.4 \\
\hline Neutrophils (\%) & 55 & 73.9 & 62 & 78 & 0.001 & 65 & 67 & 54.5 & 72 \\
\hline Eosinophils (\%) & 3.5 & 2 & 3 & 2.7 & ns & 2 & 4 & 3 & 2.6 \\
\hline $\mathrm{Hb}(\mathrm{g} / \mathrm{dL})$ & 12.7 & 11 & 10.9 & 10.7 & 0.029 & 11 & 9.2 & 13.8 & 11.1 \\
\hline $\operatorname{PLT}\left(\times 10^{3} / \mathrm{mm}^{3}\right)$ & 342 & 394 & 440 & 385 & ns & 383 & 422 & 301 & 400 \\
\hline ESR (mm 1st h) & 14 & 63 & 80 & 118 & 0.007 & 66 & 45 & 13 & 70 \\
\hline CRP (mg/dL) & 20 & 57 & 80 & 110 & ns & 57 & - & 27 & 74 \\
\hline $\mathrm{LDH}(\mathrm{mg} / \mathrm{dL})$ & 498 & 319 & 380 & 404 & ns & 324 & - & 446 & 381 \\
\hline
\end{tabular}

${ }^{*}$ number of patients, ${ }^{* *}$ median values, ${ }^{* * *}$ MC: mixed cellularity, LD: lymphocytic depletion, LP: lymphocytic predominance, NS: nodular sclerosis, ${ }^{*}$ nonsignificant.

TABLE 3: Characteristics of patients with relapse.

\begin{tabular}{|c|c|c|c|c|c|c|c|c|c|c|c|c|}
\hline \multirow{2}{*}{\multicolumn{2}{|c|}{ Time }} & \multirow{2}{*}{ Gender } & \multirow{2}{*}{ Age (years) } & \multirow{2}{*}{ Initial stage } & \multirow{2}{*}{ Histology } & \multirow{2}{*}{ Treatment adequate } & \multirow{2}{*}{ Treatment } & \multirow{2}{*}{$\mathrm{CT}$} & \multicolumn{2}{|l|}{ RT } & \multirow{2}{*}{ Outcome } & \multirow{2}{*}{ Time } \\
\hline & & & & & & & & & Adequate & Dose & & \\
\hline 1 & $20^{*}$ & Girl & 13.5 & IV & NS & yes & CT \& RT & $\mathrm{OPPA}+\mathrm{COPP}$ & yes & $20 \mathrm{~Gy}$ & NED & $83^{*}$ \\
\hline 2 & 24 & Boy & 8 & II & $\mathrm{MC}$ & yes & CT \& RT & VBVP & yes & $20 \mathrm{~Gy}$ & NED & 101 \\
\hline 3 & 30 & Boy & 13.2 & III & NS & yes & CT \& RT & ABVD + MOPP & yes & $20 \mathrm{~Gy}$ & NED & 175 \\
\hline 4 & 25 & Boy & 6 & IV & NS & no & CT \& RT & ABVD + MOPP & yes & $20 \mathrm{~Gy}$ & NED & 274 \\
\hline 5 & 65 & Boy & 14 & IV & LP & no & CT \& RT & OPPA + COPP & no & $20 \mathrm{~Gy}$ & NED & 129 \\
\hline
\end{tabular}

${ }^{*}$ months from diagnosis, MC: mixed cellularity, LD: lymphopenic depletion, LP: lymphocytic predominant, NS: nodular sclerosis.

CT: chemotherapy, RT: radiotherapy, NED: no evidence of disease.

infiltration of liver, spleen, and kidney in 3,14 , and 1 pts respectively.

3.2. Clinical Staging. Seven pts (12\%) were classified as stage I disease, 25 (43.1\%) as stage II (IIA: 12, IIB: 13), 15 (25.9\%) as stage III (IIIA: 3, IIIB: 12), and $11(19 \%)$ as stage IV (IVA: 5, IVB: 6). Lung infiltration was detected in 3 and bone disease in 2. Lymphangiogram revealed abnormal findings in one of 9 pts having the examination performed whereas abnormal bone scanning and bone marrow involvement was found each in 2.

3.3. Histology Subtypes. Nodural sclerosis was the most common subtype (40/58, 69\%). Mixed cellularity subtype was found more frequently in stages II and III, nodular sclerosis in stage II whereas the child with lymphocyte depletion subtype had advanced disease (stage IV). In Table 2 the correlation between histology subtype and stage of disease with epidemiologic and laboratory findings is shown.

3.4. Treatment Periods. Twenty-one and 37 patients with HL were treated during the first (1987 to 1993) and the second period (1994 to 2006), respectively.
3.5. Response and Outcome. Complete Remission (CR) was achieved in all patients and both chemotherapy and radiotherapy were well tolerated and without significant toxicity. All chemotherapy was given as outpatient therapy. Patients received full doses of chemotherapy with occasional delays for low blood counts. Patients experienced occasional myelosuppression, mucositis, and nausea managed by supportive care. Fifty-two pts live in their first complete remission with a DFS (disease free survival) of 90\%. In $5(8.6 \%)$ relapse occurred 20-65 months from diagnosis (median 25). All received salvage aggressive chemotherapeutic treatment in combination with high dose CT and autologous stem-cell transplantation (SCT). Additional radiotherapy was given to involved areas using individualized doses in two of them. Characteristics of pts with relapse are shown in Table 3. Of the five relapsed all live in second CR 63-249 months (median 117) from the diagnosis of the relapse and 83274 months (median 141) from the diagnosis of the disease. Three pts developed thyroid cancer, 71-144 months after 20 Gy to a field that included the thyroid gland, presumably radiation induced. All were treated and believed to be cured after subtotal thyroidectomy and $\left({ }^{131} \mathrm{I}\right)$ thyroid ablation. Unfortunately the third patient developed osteosarcoma as third malignancy 94 months after primary treatment, outside and unrelated to her prior radiation field. She died of refractory metastatic disease. Characteristics of pts who developed second and third malignancy are presented in Table 4. With a median followup of 187 months (range, 
TABLE 4: Characteristics of patients with second or third malignancy.

\begin{tabular}{|c|c|c|c|}
\hline & 1 & 2 & 3 \\
\hline $\begin{array}{l}\text { Time from } \\
\text { Dx }\end{array}$ & 144 & 48 & 71 \\
\hline Gender & Boy & Girl & Girl \\
\hline $\begin{array}{l}\text { Age at Dx } \\
\text { (years) }\end{array}$ & 4.9 & 12.2 & 11.2 \\
\hline Initial stage & I & II & III \\
\hline $\begin{array}{l}\text { Histologic } \\
\text { subtype }\end{array}$ & NS & NS & NS \\
\hline $\begin{array}{l}\text { Treatment } \\
\text { adequate }\end{array}$ & Yes & Yes & Yes \\
\hline Treatment & CT \& RT & CT \& RT & CT \& RT \\
\hline $\mathrm{CT}$ & ABVD & $\mathrm{VBVP}+\mathrm{OPPA}$ & $\mathrm{ABVD}+\mathrm{MOPP}$ \\
\hline \multicolumn{4}{|l|}{ Radiotherapy } \\
\hline $\begin{array}{l}\text { In initially } \\
\text { irradiated } \\
\text { field }\end{array}$ & Yes & Yes & Yes \\
\hline Dose & 20 Gy & 20 Gy & 20 Gy \\
\hline Outcome & NED & NED & Deceased \\
\hline Time & $229^{*}$ & 112 & - \\
\hline
\end{tabular}

* months from diagnosis, MC: mixed cellularity, LD: lymphocyte depletion, LP: lymphocytic predominant, NS: nodular sclerosis.

CT: chemotherapy, RT: radiotherapy, NED: no evidence of disease.

(1) Thyroid cancer.

(2) Thyroid cancer.

(3) Thyroid cancer (2nd) and Osteosarcoma (3rd) 94 months from diagnosis.

67 to 306 ), the overall survival rate is $98 \%$ and the eventfree survival $86.2 \%$ (50/58 pts). The correlation between epidemiologic, clinical, imaging, laboratory, and treatment parameters is shown in Table 5.

\section{Discussion}

The third most common cancer in children $\leq 15$ years of age is lymphoma with Hodgkin accounting for $40 \%$. The recent World Health Organization classification adopted the Revised European-American Classification of Lymphoid Neoplasms, which classifies HL into two disease entities according to recent clinical and biologic data: the classical Hodgkin Lymphoma (CHL, 95\% of cases) which incorporates the four histology subtypes: nodular sclerosis (NS), mixed cellularity (MC), lymphocyte-depleted (LD), and lymphocyte-rich (LR) and the Nodular lymphocyte predominant Hodgkin lymphoma (NLPHL, 5\% of cases) which is a distinct entity compared to classical disease [11]. In children, mixed cellularity, nodular lymphocyte predominant, and nodular sclerosis are the subtypes more commonly seen. Patients with NLPHL usually present with early stage, cervical or inguinal involvement, are predominantly male and most frequently 25-45 years old [12]. In CHL lymphocyte predominant and nodular sclerosis subtype correlates with early-stage disease, whereas lymphocyte depletion subtype with advanced stage and mixed cellularity with intermediate stage [13]. All our patients had classical HL with nodular sclerosis subtype being the most common (69\%). Analysis of data showed correlation of histology subtype with the gender. No correlation of histology to outcome was found in agreement to the findings of other studies [14]. It is obvious that after the introduction of current treatment modalities, histology has less important prognostic significance. Between industrialized and developing countries a difference concerning the age of patients is noted $[15,16]$.

The median age of our patients was 12 years, whereas the number of children with age of 5 years or less was of importance ( $5 \%, 3$ patients). Regarding gender, the literature reports a slight male predominance $(1.5: 1)$ but as pts grow, males and females are equally involved [3]. In this study as well, we detected a predominance of males whereas girls were diagnosed at a significant older age.

HL in childhood has been shown to be related to various factors such as higher socioeconomic status in industrialized countries, viral infections (EBV, HHV-6, CMV), and immunodeficiency [3]. A significant proportion of patients with HL have elevated antibody titers to EBV [17]. In Western countries the correlation between disease and EBV infection exceeds 50\% [18]. Our patients had EBVIgG antibodies in $72.5 \%$ as do many healthy children and adolescents in our country. HL has also been associated with autoimmune diseases [19].

Presence of B-symptoms (unexplained fever with temperature above $38^{\circ} \mathrm{C}$, drenching night sweats, weight loss of $10 \%$ ) is considered to be of prognostic significance and correlate with more aggressive disease [20]. Among our patients nonspecific general symptoms were documented in $31 \%$ rising to $46 \%$ in pts with stage III or IV. Nevertheless, no correlation was found between the presence of B-symptoms and outcome of our patients as also shown by others [5]. On the contrary, other investigators have shown unfavorable prognosis among patients with B-symptoms [13].

Also in our study, outcome of patients was not related to laboratory findings at diagnosis as leukocytosis, lymphopenia, eosinophilia, anemia $(<10.5 \mathrm{~g} / \mathrm{dL})$, elevated ESR $(>70 \mathrm{~mm} / \mathrm{h}$ ) high LDH. Moreover, in the present study, we could not detect any correlation between imaging findings (bulky mediastinal disease) and outcome. However, in the literature this was correlated with advanced disease, worse prognosis and requires more aggressive treatment [21]. It has also been shown that patients with hemoglobin of $11 \mathrm{~g} / \mathrm{dL}$ or less, mediastinal bulky disease, advanced stage, presence of Bsymptoms, age greater than 7 years, extranodal disease, and greater number of involved nodal sites had worse prognosis [4-6, 12-14]. Nevertheless, the lack of prognostic factors detected in our study may be attributed to the small study population combined with the very small number of events.

The vast majority of children with HL nowadays have an excellent chance of definite cure. The high curative rates as well as the prevention of late effects (cardiac toxicity, endocrine sequelae, bone growth alterations, pulmonary sequelae, and secondary malignancy) have to be among primary goals when managing children with HL [22]. During the last twenty-five years 58 children in our institution were 
Table 5: Prognostic factors for the outcome.

\begin{tabular}{|c|c|c|c|c|c|c|c|c|}
\hline & \multirow{2}{*}{ Patients } & \multirow{2}{*}{$\%$} & \multicolumn{3}{|c|}{$\%$} & \multicolumn{3}{|c|}{$P$} \\
\hline & & & DFS & EFS & OS & DFS & EFS & OS \\
\hline \multicolumn{9}{|l|}{ Age (years) } \\
\hline$\leq 8$ & 9 & 15.5 & 77.8 & 66.7 & 100 & \multirow{2}{*}{ ns } & \multirow{2}{*}{ ns } & \multirow{2}{*}{ ns } \\
\hline$>8$ & 49 & 84.5 & 91.8 & 89.8 & 98 & & & \\
\hline \multicolumn{9}{|l|}{ Gender } \\
\hline Boys & 35 & 60 & 88.6 & 85.7 & 100 & \multirow{2}{*}{ ns } & \multirow{2}{*}{ ns } & \multirow{2}{*}{ ns } \\
\hline Girls & 23 & 40 & 91.3 & 87 & 95.7 & & & \\
\hline \multicolumn{9}{|l|}{ Stage } \\
\hline I & 7 & 12 & 100 & 85.7 & 100 & \multirow{4}{*}{ ns } & \multirow{4}{*}{ ns } & \multirow{4}{*}{ ns } \\
\hline II & 25 & 43 & 92 & 92 & 96 & & & \\
\hline III & 15 & 26 & 93.3 & 86.7 & 100 & & & \\
\hline IV & 11 & 19 & 72.7 & 72.7 & 100 & & & \\
\hline \multicolumn{9}{|l|}{ Histologic subtype } \\
\hline Mixed cellularity & 13 & 22 & 92.3 & 92.3 & 100 & \multirow{4}{*}{$\mathrm{ns}$} & \multirow{4}{*}{ ns } & \multirow{4}{*}{ ns } \\
\hline Lymphopenic depletion & 1 & 2 & 100 & 100 & 100 & & & \\
\hline Lymphocytic predominant & 4 & 7 & 75 & 75 & 100 & & & \\
\hline Nodular sclerosis & 40 & 69 & 90 & 85 & 97.5 & & & \\
\hline \multicolumn{9}{|l|}{ B-symptoms } \\
\hline Present & 18 & 31 & 94.4 & 94.4 & 100 & \multirow{2}{*}{ ns } & \multirow{2}{*}{ ns } & \multirow{2}{*}{ ns } \\
\hline Absent & 40 & 69 & 87.5 & 82.5 & 97.5 & & & \\
\hline \multicolumn{9}{|l|}{ Mediastinum } \\
\hline Great widening & 12 & 21 & 91.7 & 83.3 & 100 & \multirow{3}{*}{ ns } & \multirow{3}{*}{ ns } & \multirow{3}{*}{ ns } \\
\hline Intermediate widening & 33 & 57 & 87.9 & 87.9 & 97 & & & \\
\hline Normal & 13 & 22 & 92.3 & 84.6 & 100 & & & \\
\hline White blood cells $\left(\times 10^{3} / \mathrm{mm}^{3}\right.$ & & & & & & & & \\
\hline$\leq 10.4$ & 29 & 51 & 93.1 & 89.7 & 100 & ns & ns & ns \\
\hline$>10.4$ & 28 & 49 & 89.3 & 85.7 & 96.4 & 110 & 110 & 110 \\
\hline Hemoglobin $(\mathrm{g} / \mathrm{dL})^{*}$ & & & & & & & & \\
\hline$\leq 11$ & 29 & 51 & 89.6 & 82.8 & 96.5 & ns & ns & $\mathrm{ns}$ \\
\hline$>11$ & 28 & 49 & 92.9 & 92.9 & 100 & 110 & 110 & \\
\hline $\operatorname{ESR}(\mathrm{mm} \mathrm{1st} \mathrm{h)*}$ & & & & & & & & \\
\hline$\leq 63$ & 29 & 51 & 93.1 & 89.7 & 100 & ns & $\mathrm{ns}$ & ns \\
\hline$>63$ & 28 & 49 & 89.3 & 85.7 & 96.4 & 110 & 110 & 110 \\
\hline
\end{tabular}

${ }^{*}$ no values for one patient.

treated with the combined use of chemotherapy and lowdose involved field radiotherapy. In 1987 we adopted the treatment approach of the SFOP MDH82 protocol [9] in order to reduce the therapy-related morbidity and mortality due to high-dose extended field irradiation, staging laparotomy, and toxic chemotherapy which were the treatment strategy before 1980s in adults and children. Later in 1994 we substituted ABVD with a less toxic combination VBVP for early-stage HL as per the MDH90 protocol [10].

In 1990s it became clear that the majority of children with clinical stages I and II can be cured without alkylating agents accused for second malignancies and sterility, and anthracyclines accused for cardiac toxicity. With this treatment policy over the last 20 years our patients had an excellent outcome. With an overall survival rate of $98 \%$ and an event-free survival of $86.2 \%$ it becomes evident that less toxic chemotherapy regimens and low-dose involved-field radiotherapy can effectively treat the majority of children with HL. The reported survival rate after relapse ranges between 50 to $80 \%$. Relapses of Hodgkin lymphoma occur more frequently during the first 3 years but some patients experienced very late relapses (10 years) [23]. For this group of pts aggressive chemotherapy followed by ASCT has shown to improve survival $[24,25]$. In our cohort of pts there have been 5 cases of disease relapse; all are disease free after salvage therapy with a median followup after relapse of 117 months (range 63 to 249).

Although we did not present toxicity data for our pts, our impression for late effects is encouraging. There has been a small number with documented abnormal cardiopulmonary testing but none require ongoing medical management and all show good exercise tolerance. Hypothyroidism was 
detected in about 30\% of our survivors, requiring only replacement hormone therapy. Gonadotoxicity is an important late effect in the management of HL but we do not have enough data to anticipate that gonadal function is substantially influenced. Second malignancies are attributed to both radiation therapy and chemotherapy. Alkylating agents and topoisomerase II inhibitors appear to be highly mutagenic [26]. Among our patients, three developed second (thyroid cancer) and one of them a third malignancy (osteosarcoma). At the time of data analysis (end of 2011), there have been no cases of secondary leukaemia and no cases of breast cancer; however, the risk increases steadily with extended followup and continuing surveillance of our patients for secondary malignancies will determine whether reduced-intensity treatment has effectively decreased their incidence.

Presently, Hodgkin lymphoma in children can be cured in at least $80 \%$ of pts. Accumulating evidence suggests that the vast majority of children with HL can be cured with the minimal required therapy by reducing the number of cycles and number of agents and using drugs with low risk of infertility, cardiopulmonary dysfunction, or second malignancies [27]. The new treatment strategies will be less toxic for favorable cases (without alkylating agents and anthracyclines) and more effective for high risk patients. The treatment of patients with relapsed HL depends on previous therapies with radiotherapy, chemotherapy, or high-dose chemotherapy followed by autologous SCT [28]. In conclusion combined-modality therapy remains the standard of care for children and adolescents with HL. However, there may be a significant number of children and adolescents who can be cured with chemotherapy alone. On the other hand, using LD-IFRT in conjunction with decreased intensity CT may produce the best results for certain patients [29].

At this moment the main question is "who can be cured without RT?" In ongoing trials positron emission tomography (PET) and especially 18-fluoro-2-deoxyglucose PET (FDG-PET) plus computed tomography (PET-CT) is increasingly used to determine response to initial CT, complete versus partial response and to detect relapse [30]. A FDG-PET scan after 2 cycles of chemotherapy may help in selecting good-prognosis HL pts who with less intensive and less morbid chemotherapy and without radiotherapy can be cured.

\section{References}

[1] J. Gurney and M. Bondy, "Epidemiology of childhood cancer," in Principles and Practice of Pediatric Oncology, A. P. Pizzo and D. G. Poplack, Eds., chapter 1, pp. 1-13, Lippincott-Raven, Philadelphia, Pa, USA, 5th edition, 2006.

[2] E. T. Petridou, A. Pourtsidis, N. Dessypris et al., "Childhood leukaemias and lymphomas in Greece (1996-2006): a nationwide registration study," Archives of Disease in Childhood, vol. 93, no. 12, pp. 1027-1032, 2008.

[3] M. Hudson, M. Onciu, and S. Donaldson, "Hodgkin's disease," in Principles and Practise of Pediatric Oncology, P. Pizzo and D. Poplack, Eds., pp. 695-721, Lippincott-Raven, Philadelphia, $\mathrm{Pa}$, USA, 5th edition, 2006.
[4] S. S. Donaldson, M. P. Link, H. J. Weinstein et al., "Final results of a prospective clinical trial with VAMP and low-dose involved-field radiation for children with low-risk Hodgkin's disease," Journal of Clinical Oncology, vol. 25, no. 3, pp. 332337, 2007.

[5] A. Oguz, C. Karadeniz, F. V. Okur et al., "Prognostic factors and treatment outcome in childhood Hodgkin disease," Pediatric Blood and Cancer, vol. 45, no. 5, pp. 670-675, 2005.

[6] M. M. Hudson, M. Krasin, M. P. Link et al., "Risk-adapted, combined-modality therapy with VAMP/COP and responsebased, involved-field radiation for unfavorable pediatric Hodgkin's disease," Journal of Clinical Oncology, vol. 22, no. 22, pp. 4541-4550, 2004.

[7] T. A. Lister, D. Crowther, S. B. Sutcliffe et al., "Report of a committee convened to discuss the evaluation and staging of patients with Hodgkin's disease: cotswolds meeting," Journal of Clinical Oncology, vol. 7, no. 11, pp. 1630-1636, 1989.

[8] E. L. Weinshel and B. A. Peterson, "Hodgkin's disease," $\mathrm{Ca}-\mathrm{A}$ Cancer Journal for Clinicians, vol. 43, no. 6, pp. 327-346, 1993.

[9] O. Oberlin, G. Leverger, H. Pacquement et al., "Low-dose radiation therapy and reduced chemotherapy in childhood Hodgkin's disease: the experience of the French Society of pediatric oncology," Journal of Clinical Oncology, vol. 10, no. 10, pp. 1602-1608, 1992.

[10] J. Landman-Parker, H. Pacquement, T. Leblanc et al., "Localized childhood Hodgkin's disease: response-adapted chemotherapy with etoposide, bleomycin, vinblastine, and prednisone before low-dose radiation therapy-results of the French society of pediatric oncology study MDH90," Journal of Clinical Oncology, vol. 18, no. 7, pp. 1500-1507, 2000.

[11] A. L. Feldman, S. Pittaluga, and E. S. Jaffe, "Classification and histopathology of the lymphomas," in The Lymphomas, G. P. Canellos, T. A. Lister, and B. Young, Eds., pp. 2-25, Saunders Elsevier, Philadelphia, Pa, USA, 2nd edition, 2006.

[12] L. Nogová, T. Rudiger, and A. Engert, "Biology, clinical course and management of nodular lymphocyte-predominant hodgkin lymphoma," Hematology, pp. 266-272, 2006.

[13] R. S. Smith, Q. Chen, M. M. Hudson et al., "Prognostic factors for children with Hodgkin's disease treated with combinedmodality therapy," Journal of Clinical Oncology, vol. 21, no. 10, pp. 2026-2033, 2003.

[14] C. L. Schwartz, "Prognostic factors in pediatric Hodgkin disease," Current Oncology Reports, vol. 5, no. 6, pp. 498-504, 2003.

[15] A. Belgaumi, A. Al-Kofide, N. Joseph, R. Jamil-Malik, Y. Khafaga, and R. Sabbah, "Hodgkin lymphoma in very young children: clinical characteristics and outcome of treatment," Leukemia and Lymphoma, vol. 49, no. 5, pp. 910-916, 2008.

[16] G. A. Viani, M. S. Castilho, P. E. Novaes et al., "Chemotherapy followed by low dose radiotherapy in childhood Hodgkin's disease: retrospective analysis of results and prognostic factors," Radiation Oncology, vol. 1, pp. 38-45, 2006.

[17] V. Dinand, R. Dawar, L. S. Arya, R. Unni, B. Mohanty, and R. Singh, "Hodgkin's lymphoma in Indian children: prevalence and significance of Epstein-Barr virus detection in Hodgkin's and Reed-Sternberg cells," European Journal of Cancer, vol. 43, no. 1, pp. 161-168, 2007.

[18] T. Portis, P. Dyck, and R. Longnecker, "Epstein-Barr Virus (EBV) LMP2A induces alterations in gene transcription similar to those observed in Reed-Sternberg cells of Hodgkin lymphoma," Blood, vol. 102, no. 12, pp. 4166-4178, 2003.

[19] O. Landgren, E. A. Engels, R. M. Pfeiffer et al., "Autoimmunity and susceptibility to Hodgkin lymphoma: a population-based 
case-control study in Scandinavia," Journal of the National Cancer Institute, vol. 98, no. 18, pp. 1321-1330, 2006.

[20] C. L. Schwartz, "The management of Hodgkin disease in the young child," Current Opinion in Pediatrics, vol. 15, no. 1, pp. 10-16, 2003.

[21] V. Diehl, H. Stein, M. Hummel, R. Zollinger, and J. M. Connors, "Hodgkin's lymphoma: biology and treatment strategies for primary, refractory, and relapsed disease," Hematology, pp. 225-247, 2003.

[22] N. L. Bartlett, "Modern treatment of Hodgkin lymphoma," Current Opinion in Hematology, vol. 15, no. 4, pp. 408-414, 2008.

[23] A. F. Cashen and N. L. Bartlett, "Therapy of relapsed hodgkin lymphoma,” Blood Reviews, vol. 21, no. 5, pp. 233-243, 2007.

[24] M. B. Bradley and M. S. Cairo, "Stem cell transplantation for pediatric lymphoma: past, present and future," Bone Marrow Transplantation, vol. 41, no. 2, pp. 149-158, 2008.

[25] E. Brusamolino and A. M. Carella, "Treatment of refractory and relapsed Hodgkin's lymphoma: facts and perspectives," Haematologica, vol. 92, no. 1, pp. 6-10, 2007.

[26] A. K. Ng and P. M. Mauch, "The impact of treatment on the risk of second malignancy after Hodgkin's disease," Annals of Oncology, vol. 17, no. 12, pp. 1727-1729, 2006.

[27] M. R. Olson and S. S. Donaldson, "Treatment of pediatric hodgkin Lymphoma," Current Treatment Options in Oncology, vol. 9, no. 1, pp. 81-94, 2008.

[28] M. Fuchs, V. Diehl, and D. Re, "Current strategies and new approaches in the treatment of Hodgkin's lymphoma," Pathobiology, vol. 73, no. 3, pp. 126-140, 2006.

[29] J. B. Nachman, R. Sposto, P. Herzog et al., "Randomized comparison of low-dose involved-field radiotherapy and no radiotherapy for children with Hodgkin's disease who achieve a complete response to chemotherapy," Journal of Clinical Oncology, vol. 20, no. 18, pp. 3765-3771, 2002.

[30] M. E. Juweid, "Utility of positron emission tomography (PET) scanning in managing patients with Hodgkin lymphoma," Hematology, pp. 259-265, 2006. 


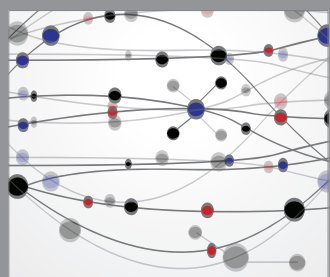

The Scientific World Journal
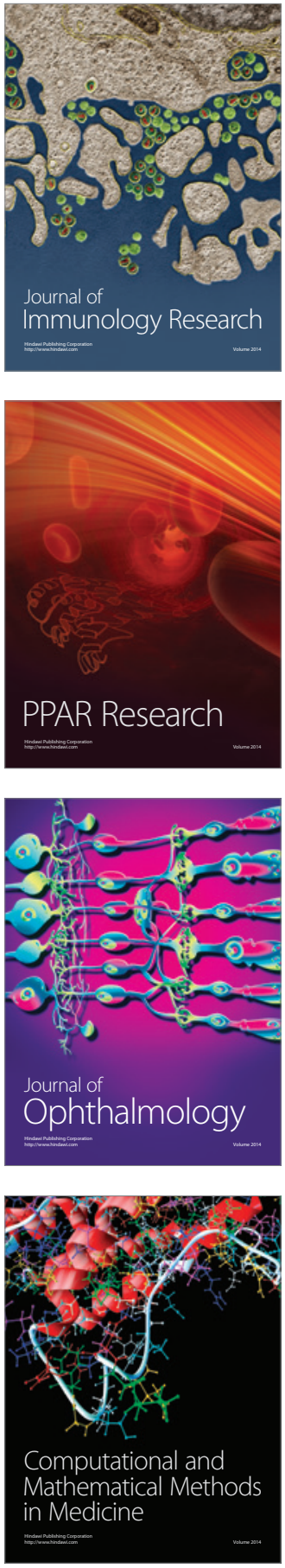

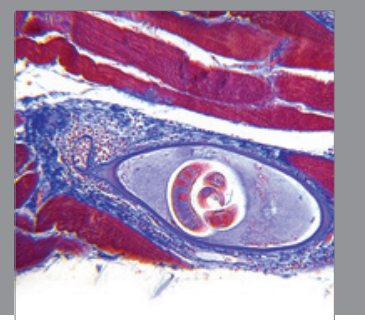

Gastroenterology

Research and Practice
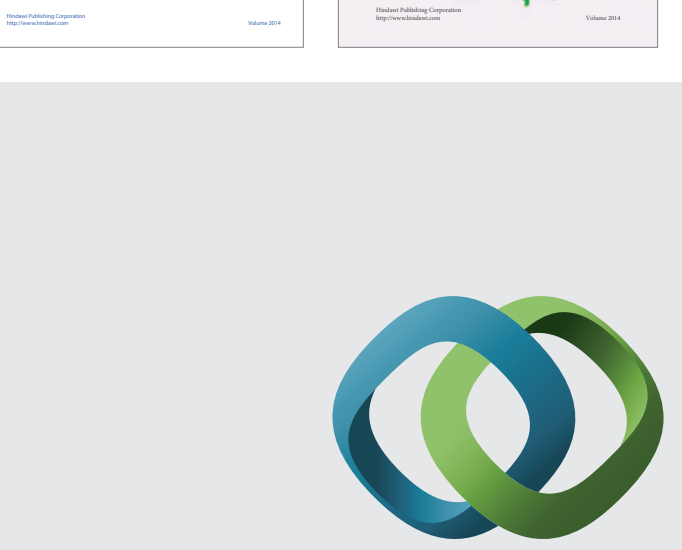

\section{Hindawi}

Submit your manuscripts at

http://www.hindawi.com
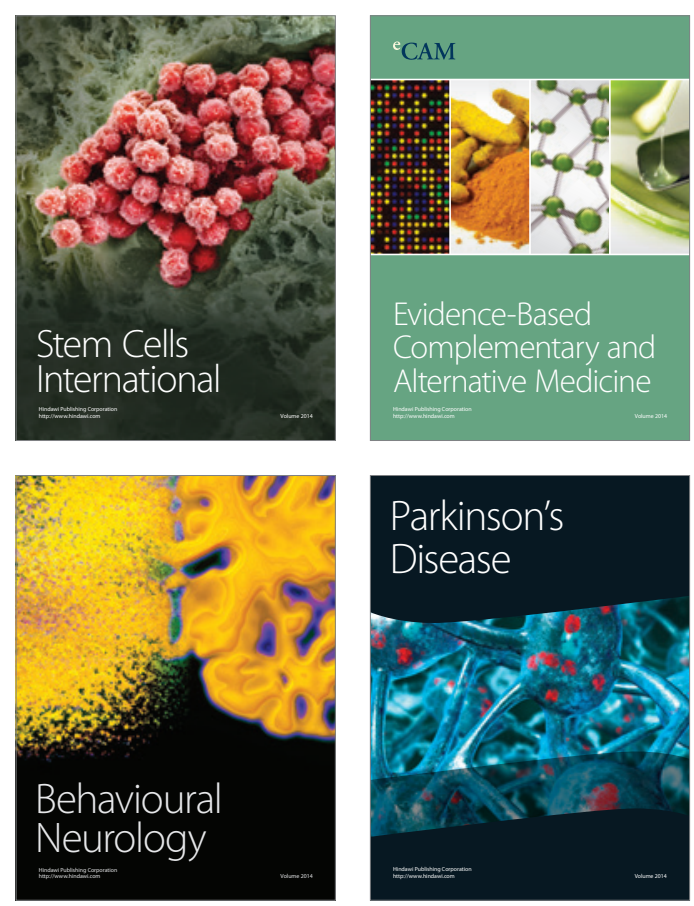

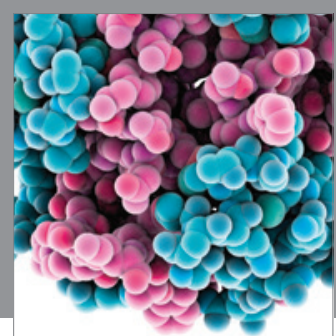

Journal of
Diabetes Research

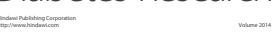

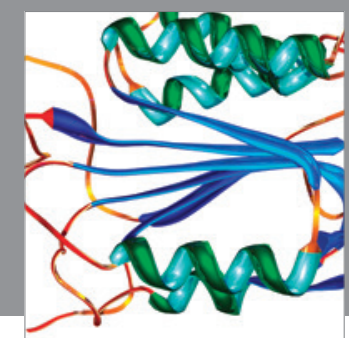

Disease Markers
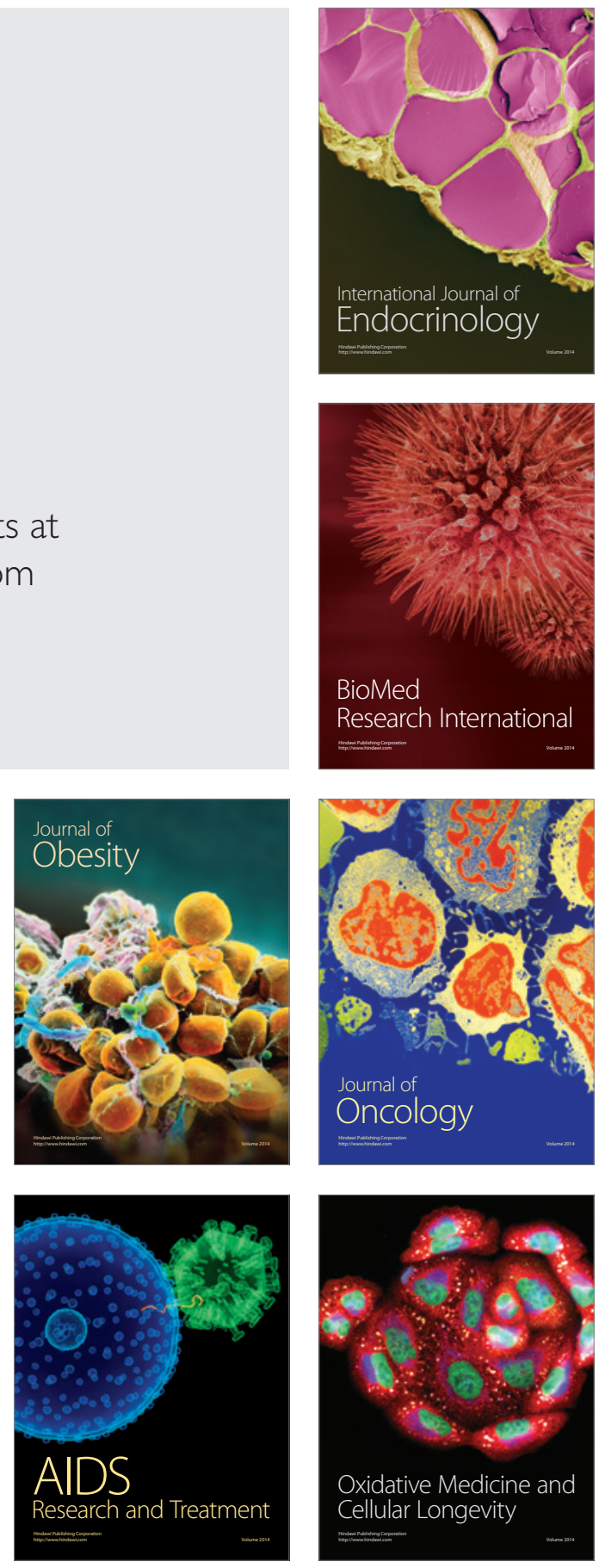